\title{
Mesurer la santé mentale positive au Canada : validation des concepts du Continuum de santé mentale - Questionnaire abrégé
}

\author{
Heather Orpana, Ph. D. (1,2); Julie Vachon, M. Sc. (1); Jennifer Dykxhoorn, M. Sc. (3); Gayatri Jayaraman, Ph. D. (1,4)
}

Cet article a fait l'objet d'une évaluation par les pairs.

Diffuser cet article sur Twitter

\section{Résumé}

Introduction : La santé mentale positive est de plus en plus reconnue comme un élément central des politiques et des programmes en matière de santé publique. On a déterminé au Canada que le Continuum de santé mentale - Questionnaire abrégé (CSM-QA) constituait un outil de mesure prometteur qu'il fallait intégrer aux enquêtes d'évaluation de la santé mentale positive de la population. Ce questionnaire vise à évaluer un modèle à trois facteurs de santé mentale positive : le bien-être émotionnel, le bien-être social et le bienêtre psychologique. Cette étude a pour but de déterminer si le CSM-QA offre une mesure adéquate de la santé mentale positive des Canadiens adultes.

Méthodologie : Nous avons réalisé une analyse factorielle confirmatoire (AFC) à l'aide des données de l'Enquête sur la santé dans les collectivités canadiennes - Santé mentale de 2012 (ESCC-SM). Nous avons également effectué une validation croisée du modèle à l'aide des données de l'Enquête sur la santé dans les collectivités canadiennes Composante annuelle (ESCC - Composante annuelle) de 2011-2012. Nous avons examiné la validité reliée à un critère dans le cadre d'analyses de corrélation entre les scores des sous-échelles du CSM-QA et les concepts positifs (comme la satisfaction à l'égard de la vie) et négatifs (comme la détresse psychologique) associés.

Résultats : Nous avons confirmé la validité du modèle à trois facteurs du bien-être émotionnel, social et psychologique dans le cadre d'une AFC de deux échantillons distincts, et ce, après avoir intégré les quatre erreurs corrélées entre les questions relatives à l'échelle du bien-être social. Nous avons observé, comme prévu, des corrélations significatives entre le bien-être émotionnel, psychologique et social et les concepts associés. Le coefficient alpha de Cronbach était de 0,82 pour les sous-échelles du bien-être émotionnel et du bien-être psychologique et de 0,77 pour le bien-être social.

Conclusion : Notre étude suggère que le CSM-QA permet de rendre compte du modèle à trois facteurs de la santé mentale positive de la population canadienne. Toutefois, la prudence est de mise lorsque l'on utilise l'échelle du bien-être social, qui ne s'est pas avérée aussi efficace que les autres facteurs, comme l'ont mis en évidence la nécessité d'ajouter plusieurs termes d'erreur corrélés afin d'obtenir un ajustement adéquat du modèle, le pourcentage plus élevé de données manquantes pour ces questions et les corrélations plus faibles avec les concepts connexes. Comme le bien-être social constitue un élément important d'une évaluation exhaustive de la santé mentale positive, la tenue d'autres recherches est recommandée.

Mots-clés : santé mentale, santé mentale positive, enquêtes et questionnaires, analyse factorielle

\section{Points saillants}

- On peut mesurer la santé mentale positive à l'aide d'un modèle à trois facteurs reposant sur le bien-être émotionnel, le bien-être psychologique et le bien-être social.

- Si l'on a étayé le modèle à trois facteurs, le facteur relatif au bienêtre social ne s'est pas avéré aussi efficace que les deux autres facteurs.

- D’autres recherches sont nécessaires afin de mettre au point une mesure plus solide et plus fiable du bien-être social en contexte canadien.

\section{Introduction}

On reconnaît de plus en plus qu'une bonne santé ne se traduit pas simplement par une " absence de maladies " et que la santé physique comme la santé mentale sont essentielles à un bien-être complet. Dans ce contexte, le rôle de la santé mentale retient de plus en plus l'attention, car elle est associée à un meilleur fonctionnement, une meilleure santé physique et une capacité accrue à contribuer à la société ${ }^{1}$. L’Agence de la santé publique du Canada (ASPC) définit la santé mentale comme : « [...] la capacité qu'a chacun d'entre nous de ressentir, de penser et d'agir de manière à améliorer notre aptitude à jouir de la vie et à relever les défis auxquels nous sommes confrontés. Il s'agit d'un sentiment positif de bien-être émotionnel et spirituel qui respecte l'importance de la culture, de l'équité, de la justice sociale, des interactions et de la dignité personnelle $»^{2}$, p.3. Cette définition est similaire à celle de

Rattachement des auteurs :

1. Agence de la santé publique du Canada, Ottawa (Ontario), Canada

2. École de psychologie, Université d'Ottawa, Ottawa (Ontario), Canada

3. Division of Psychiatry, University College London, Londres, Royaume-Uni

4. Département d'épidémiologie et de médecine communautaire, Université d'Ottawa, Ottawa (Ontario), Canada

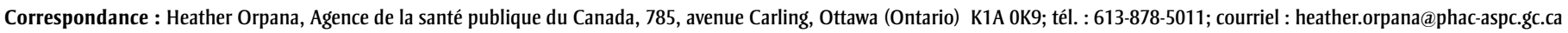


l'Organisation mondiale de la santé (OMS), selon laquelle "La santé mentale est un état de bien-être dans lequel une personne peut se réaliser, surmonter les tensions normales de la vie, accomplir un travail productif et contribuer à la vie de sa communauté $»^{3}$.

Un certain nombre de mesures de la santé mentale positive ont déjà été mises au point. On compte parmi elles l'échelle de bien-être mental de Warwick et Edinburgh (WEMWBS) ${ }^{4}$, l'échelle d'épanouissement de Diener (Diener's Flourishing Scale) ${ }^{5}$, l'échelle de Huppert et de $\mathrm{So}^{6}$, le profileur PERMA (émotions positives, engagement, relations positives, signification et accomplissements) basé sur le modèle de psychologie positive de Seligman ${ }^{7}$ et le Continuum de santé mentale (CSM) de Keyes $^{8,9}$. La majorité d'entre elles ont été élaborées dans le but de comprendre les différences entre individus en matière de bien-être, à l'exception de la WEMWBS, spécialement mise au point pour assurer un suivi du bien-être de la population.

Le CSM est une mesure de la santé mentale positive élaborée par Keyes ${ }^{8}$ à partir d'un modèle à trois facteurs de bien-être : le bien-être émotionnel, le bien-être psychologique et le bien-être social. Le volet sur le bien-être émotionnel vise à évaluer les émotions positives, la satisfaction à l'égard de la vie et l'intérêt pour la vie et s'appuie sur les travaux de Bradburn sur les émotions positives ${ }^{10}$ et ceux de Cantril ${ }^{11}$ sur la satisfaction à l'égard de la vie. Le volet sur le bien-être psychologique évalue le fonctionnement dans six domaines définis par $\operatorname{Ryff}^{12}$, soit l'autonomie, la maîtrise, l'épanouissement personnel, les relations positives avec les autres, les buts dans la vie et l'acceptation de soi. Le volet sur le bienêtre social s'appuie quant à lui sur les travaux de Keyes et il fait état de la perception des individus à l'égard de leurs expériences en société et s'articule autour de cinq concepts : la contribution sociale, l'acceptation sociale, la cohérence sociale, la réalisation sociale et l'intégration sociale ${ }^{13}$.

L'outil CSM original comprend des souséchelles du bien-être émotionnel, du bienêtre psychologique et du bien-être social et repose sur 40 éléments. Keyes a utilisé un critère reposant sur la répartition pour déterminer un degré élevé de santé mentale positive, qualifiée de "florissante ", et attribué aux répondants ayant obtenu un score dans le tertile supérieur d'au moins l'une des échelles de bien-être émotionnel et d'au moins six des onze échelles de bien-être social et psychologique. Selon cette méthode, la santé mentale de 17,2\% de l'échantillon s'est révélée " florissante », celle de 56,6\% "modérément bonne », et celle de $12,1 \%$ " languissante ${ }^{8}$ ». Le questionnaire CSM initial étant trop long, Keyes a choisi [traduction] « les éléments les plus prototypiques qui représentaient la définition de chacune des facettes du bienêtre »", p. 1 pour créer les 14 questions du CSM-QA, largement utilisé aujourd'hui et autour duquel la présente étude s'articule. Nous ne savons pas si le jugement de l'enquêteur a été le seul critère de choix ou si certaines analyses ont contribué à orienter la sélection des questions de la version courte de l'échelle. Le CSM-QA a été mis en œuvre à la fois dans l'ESCC - Composante annuelle de 2011-2012 et dans l'ESCCSM de 2012.

Même si le CSM-QA est utilisable avec une échelle continue de scores, on l'utilise fréquemment pour déterminer la prévalence des participants dont la santé mentale est "florissante ", soit ceux ayant répondu "presque tous les jours » ou « tous les jours " à au moins l'une des trois questions sur le bien-être émotionnel et à six des onze questions sur le bien-être psychologique et social ( $"$ au moins une et au moins six »). Keyes affirme que cette méthode d'identification des sujets à la santé mentale "florissante » est similaire à la méthode normalisée d'évaluation et de diagnostic d'un épisode dépressif majeur ${ }^{8,9}$. Nous considérons toutefois qu'une approche diagnostique de maladie mentale n'est pas nécessairement pertinente pour définir des états positifs de santé mentale. De plus, avec cette méthode de définition des répondants à la santé mentale "florissante », une personne ayant des scores élevés sur le plan du bien-être psychologique seulement (six questions) et exclusivement des scores faibles en matière de bien-être social peut être considérée comme ayant une santé mentale "florissante ", ce qui est contradictoire avec la théorie selon laquelle une santé mentale positive exige des degrés élevés de bien-être à la fois émotionnel, psychologique et social.

Un certain nombre d'études ont confirmé la pertinence du modèle à trois facteurs et un fonctionnement adéquat de l'échelle du CSM-QA. Keyes et collab. ${ }^{14}$ ont réalisé une analyse factorielle confirmatoire (AFC) des réponses par échelles auprès d'un échantillon de Sud-Africains parlant le setswana et ont confirmé le modèle à trois facteurs. Lamers et collab. ${ }^{15}$ ont montré que les analyses confirmaient le modèle à trois facteurs, mais ont également conclu que la sous-échelle du bien-être social n'était que modérément fiable alors que les souséchelles du bien-être psychologique et du bien-être émotionnel présentaient une fiabilité élevée. La structure à trois facteurs a également été confirmée dans le cadre d'une étude auprès de répondants néerlandais, sud-africains et iraniens ${ }^{16}$. Toutefois, afin de parvenir à un ajustement adéquat du modèle, la covariance de certains des résidus de questions a été tolérée.

Les études ayant évalué la santé mentale florissante à l'aide de la méthode " au moins une et au moins six » ont abouti à une hiérarchie de prévalence de santé mentale florissante de 49,3\% chez les étudiants américains de niveau collégial ${ }^{17}$, $37,9 \%$ chez les jeunes Américains ${ }^{18}$, $20 \%$ au sein d'un échantillon de Sud-Africains parlant le setswana ${ }^{14}$ et $28,5 \%$ chez les Italiens adultes ${ }^{19}$. D'après cette méthode de notation du CSM-QA, la prévalence d'une santé mentale florissante chez les Canadiens était de $76,9 \%$ en $2012^{20}$. Cette prévalence nettement plus élevée au Canada que dans les autres pays a soulevé des questions quant au fonctionnement de cette échelle dans le cadre d'enquêtes auprès de la population en contexte canadien.

Cet article a pour but de décrire les spécificités des mesures du CSM-QA en contexte canadien, d'examiner sa structure factorielle et de proposer une méthode uniforme sur les plans conceptuel et empirique pour rendre compte des résultats en santé mentale positive, et ce, en fonction de l'ensemble des questions du questionnaire. La validité reliée aux critères et la cohérence interne sont également examinées. Nous avions fait l'hypothèse de corrélations positives avec les sous-échelles du CSM-QA et l'auto-évaluation de la santé mentale, la satisfaction à l'égard de la vie et le sentiment d'appartenance à la communauté. Nous avions fait l'hypothèse de corrélations négatives avec l'outil Disability Assessment Schedule de l'OMS (WHODAS) d'évaluation des handicaps de l'OMS, des interactions sociales négatives et de la détresse psychologique. Nous nous attendions aussi à ce que les coefficients alpha de Cronbach témoignent d'une cohérence interne adéquate, sans redondance des éléments, et de scores avec une moyenne de $0,8^{21}$. 


\section{Méthodologie}

\section{Données}

L'ESCC-SM de 2012 est une enquête-ménage par échantillonnage menée auprès de 25113 Canadiens âgés de 15 ans et plus provenant des 10 provinces, excluant les personnes vivant dans les réserves et autres lieux de résidence autochtones, le personnel des Forces canadiennes et les personnes vivant en établissement ${ }^{22}$, soit moins de $3 \%$ de la population canadienne. Nous avons utilisé un plan d'échantillonnage à plusieurs niveaux basé sur celui de l'Enquête sur la population active afin de nous assurer d'une représentation adéquate de la population par groupe d'âge et par sexe dans chaque province. La participation à l'enquête était volontaire. Le taux de réponse combiné (ménages et personnes) a été de $68,9 \%$. La collecte de données a eu lieu du 2 janvier 2012 au 31 décembre 2012. Les données ont été recueillies par entrevue sur place assistée par ordinateur (EPAO) et par entrevue téléphonique assistée par ordinateur (ETAO), 87 \% des entrevues ayant été réalisées en personne. Les entrevues par personne interposée n'étaient pas autorisées dans le cadre de cette enquête. Statistique Canada a calculé les poids d'échantillonnage afin de garantir que les estimations pondérées étaient représentatives de la population des ménages canadiens des 10 provinces.

L'ESCC - Composante annuelle est une enquête-ménage par échantillonnage continue menée auprès d'environ 65000 Canadiens âgés de 12 ans et plus et provenant des 10 provinces et 3 territoires et excluant les personnes vivant dans les réserves et autres lieux de résidence autochtones, le personnel à temps plein des Forces canadiennes, les personnes vivant dans les régions sanitaires québécoises du Nunavik et des Terres-Cries-de-la-Baie-James et enfin les personnes vivant en établissement $^{23}$, soit moins de $3 \%$ de la population canadienne. L'ESCC utilise trois bases d'échantillonnage pour sélectionner l'échantillon de ménages : une base aréolaire fondée sur l'Enquête sur la population active (40,5\%), une liste de numéros de téléphone $(58,5 \%)$ et une base sélectionnée par composition aléatoire (1\%). De nombreux cycles peuvent être combinés. Les données utilisées dans ces analyses ont été collectées entre le $1^{\text {er }}$ janvier 2011 et le 31 décembre 2012. Le taux de réponse combiné (ménages et personnes) en
2011-2012 a été de 68,4%. Les données ont été recueillies par l'entremise d'EPAO et d'ETAO. Statistique Canada a calculé les poids d'échantillonnage. Les données ont été obtenues auprès de Statistique Canada, et incluaient seulement les microdonnées recueillies auprès de répondants ayant accepté de partager leurs données avec l'Agence de la santé publique du Canada et Santé Canada.

\section{Mesures}

\section{Variables communes aux deux enquêtes} Santé mentale positive

Le CSM-QA est constitué de 14 questions portant sur le bien-être émotionnel, le bienêtre psychologique et le bien-être social. Toutes les questions relatives à ces éléments suivent le format suivant : "Au cours du dernier mois, à quelle fréquence vous êtes-vous senti ou avez-vous senti...? » Les choix de réponse sont " jamais ", " une ou deux fois ", " environ une fois par semaine ", " environ deux ou trois fois par semaine", " presque tous les jours » et " tous les jours". Dans l'ESCC, la sixième question est formulée comme dans le CSM-QA original : " notre société devient un meilleur endroit pour les gens comme vous ». Depuis l'ajout de cet élément à l'ESCC, Keyes a suggéré de le changer pour « la société devient un meilleur endroit pour tout le monde " ou par " notre société est un bon endroit » mais ce changement n'a pas été effectué dans les ESCC ${ }^{9}$.

La méthode de notation originale consistait à attribuer de 1 à 6 points aux catégories de réponse " jamais ", " une ou deux fois par semaine », " environ une fois par semaine », " environ deux ou trois fois par semaine ", " presque tous les jours " et " tous les jours ". Nous avons créé une méthode de notation de substitution qui constitue un reflet plus précis des mesures sous-jacentes de catégories de réponse et qui consiste à convertir le contenu sémantique de la catégorie de réponse en jours et en échelle proportionnelle. Nous avons ainsi attribué une valeur de 28 jours (4 semaines $\times 7$ jours par semaine) à " chaque jour ", de 20 jours ( 4 semaines $\times$ 5 jours par semaine) à "presque tous les jours ", de 10 jours ( 4 semaines $\times 2,5$ jours par semaine) à « environ deux ou trois fois par semaine ", de 4 jours (4 semaines $\times$ 1 jour par semaine) à " environ une fois par semaine ", de 1,5 jour à " une ou deux fois » et de 0 jour à « jamais ». Nous avons utilisé les deux méthodes de notation pour réaliser des AFC dans deux modèles distincts.

\section{Variables individuelles}

Le sexe, le groupe d'âge, la meilleure estimation possible du revenu, l'état matrimonial et l'activité professionnelle étaient tous autodéclarés, exception faite des cas de non-réponse sur le revenu pour lesquels Statistique Canada a fourni les données manquantes.

\section{Variables spécifiques à l'ESCC-SM de 2012}

Voici quelles variables ont été utilisées pour nos analyses de validité reliée aux critères.

Nous avons mesuré l'auto-évaluation de la santé mentale à l'aide d'une seule question, soit "En général, diriez-vous que votre santé mentale est : excellente? très bonne? bonne? passable? mauvaise? » Nous avons dichotomisé les réponses à cette question en "passable », " mauvaise» et « bonne » d'une part et " très bonne » et « excellente » d'autre part.

Nous avons aussi mesuré la satisfaction à l'égard de la vie à l'aide d'une seule question : " À l'aide d'une échelle de 0 à 10 , quel sentiment éprouvez-vous maintenant à l'égard de votre vie, où 0 signifie "Très insatisfait" et 10 signifie "Très satisfait"? " Cette question est conforme aux recommandations de l'Organisation de coopération et de développement économiques (OCDE) sur la satisfaction à l'égard de la vie $^{24}$.

Nous avons mesuré le sentiment d'appartenance à l'aide d'une seule question : «Comment décririez-vous votre sentiment d'appartenance à votre communauté locale? Diriez-vous qu'il est : très fort? plutôt fort? plutôt faible? très faible? »

Nous avons mesuré le soutien social à l'aide de l'Échelle de provisions sociales (Social Provisions Scale) de Cutrona et Russell $^{25}$ en utilisant dix questions pour mesurer cinq types de ressources sociales (l'attachement, l'encadrement, l'intégration sociale, les alliances fiables et la confirmation de sa valeur) sur une échelle continue de 0 à 40 .

L'échelle de détresse psychologique de Kessler (K6) a été mise au point afin de distinguer les cas de maladie mentale grave des cas d'absence de maladie, et ce, en fonction des symptômes de détresse 
psychologique non spécifiques figurant dans le National Health Interview Survey des États-Unis. Nous avons utilisé la version à six questions (K6) pour nos analyses, avec un score mesuré sur une échelle continue de 0 à $24^{26}$.

C'est la version courte 2.0 de l'outil WHODAS d'évaluation des handicaps de l'OMS à 12 questions ${ }^{27}$ qui a été utilisée dans l'ESCC-SM de 2012. Les scores varient de 0 (aucune invalidité) à 100 (invalidité complète).

Les interactions sociales négatives ont été mesurées à l'aide de quatre questions visant à évaluer l'exposition aux interactions sociales négatives à partir des travaux de Krause $^{28}$. Nous avons combiné les réponses à ces quatre questions afin d'obtenir des scores sur une échelle de 0 à 12 .

\section{Analyses statistiques}

\section{Statistiques descriptives}

Nous avons calculé les statistiques descriptives et une matrice de corrélation pour tous les éléments de l'échantillon à l'essai (ESCC-SM de 2012). Nous avons calculé les coefficients alpha de Cronbach pour l'ensemble de l'outil et les trois souséchelles. Nous avons effectué nos analyses à l'aide de la version 5.1 du SAS Enterprise Guide (SAS Institute Inc, Cary, Caroline du Nord, États-Unis). Nous avons pondéré l'ensemble des analyses afin de tenir compte du plan d'échantillonnage à plusieurs niveaux. Comme il n'était pas possible d'employer la méthode bootstrap dans le cadre de l'AFC ou de l'analyse de corrélation, nous avons utilisé des poids d'échantillonnage remis à l'échelle pour toutes les analyses.

\section{Analyse factorielle confirmatoire}

Nous avons utilisé l'ESCC-SM de 2012 pour tester le modèle original. Nous avons réalisé une AFC et une estimation de vraisemblance maximale robuste (VMR) à l'aide de la version $7.3 \mathrm{du}$ logiciel Mplus (Muthen et Muthen, Los Angeles, Californie, ÉtatsUnis). L'estimation de VMR permet d'utiliser des poids et tient également compte de la non-normalité des données en cas d'erreurs de calcul. Nous avons ensuite testé le modèle définitif avec l'échantillon de l'ESCC de 2011-2012 afin de vérifier si la structure factorielle redéfinie se confirmait avec un échantillon différent. Conscients des divergences dans la littérature quant à ce qui constitue un ajustement adéquat, nous avons opté pour les suggestions de Hu et Bentler ${ }^{29}$ : une valeur d'au moins 0,95 pour l'indice d'ajustement comparatif (IAC) et l'indice de Tucker et Lewis (ITL), une valeur de moins de 0,08 pour la racine de l'erreur quadratique moyenne normalisée (REQMN) et une valeur de moins de 0,06 pour l'erreur-type de l'approximation (ETA). Comme le logiciel Mplus ne permet pas d'intégrer des poids d'échantillonnage (bootstrap) à une AFC, nous avons décidé, après avoir consulté Statistique Canada, d'utiliser des poids remis à l'échelle, divisés par l'effet moyen du plan d'échantillonnage pour toutes les AFC.

\section{Validité reliée aux critères}

Nous avons examiné la validité reliée aux critères en utilisant des analyses de corrélation entre les scores des sous-échelles du CSM-QA et les concepts positifs et négatifs associés (auto-évaluation de la santé mentale, satisfaction à l'égard de la vie, sentiment d'appartenance, provisions sociales, détresse psychologique, interactions sociales négatives et outil WHODAS d'évaluation des handicaps de l'OMS). Comme nous présentons plusieurs corrélations dans les analyses de validation, nous avons opté pour une valeur prudente de $\mathrm{p}<0,001$, qui équivaut à une correction de Bonferroni pour 50 analyses.

\section{Résultats}

\section{Analyse factorielle confirmatoire}

L'échantillon de l'ESCC-SM de 2012 était représentatif de la population des ménages canadiens vivant dans les provinces. Environ la moitié des répondants de l'échantillon était des hommes et l'âge moyen était de 47,2 ans. Un peu plus de $75 \%$ des participants ont répondu à l'enquête en anglais, $22 \%$ ont répondu en français et moins de $1 \%$ dans une autre langue. Près de deux tiers des répondants ont affirmé éprouver un sentiment d'appartenance à leur communauté fort ou très fort. Le score moyen pour la satisfaction à l'égard de la vie était de 7,95 sur une échelle de 0 à 10 . Sept pour cent des répondants présentaient un degré de détresse psychologique sur l'échelle de Kessler (K6) supérieur ou égal à 9, ce qui correspond aux niveaux de détresse observés chez les personnes traversant un épisode dépressif ou atteintes d'un trouble anxieux $^{26}$ (données non présentées).

L'échantillon de l'ESCC de 2011-2012 était représentatif de la population des ménages canadiens vivant dans les provinces et les territoires. Les hommes comptaient pour environ la moitié de la population visée par l'enquête. L'âge moyen des répondants était de 47 ans. Si l'anglais était la langue de réponse la plus utilisée (74 \% des répondants), $21 \%$ des participants ont répondu en français et $5 \%$ ont répondu dans une autre langue (données non présentées).

\section{Réponses au Continuum de santé mentale - Questionnaire abrégé}

De façon générale, nous avons obtenu des scores élevés sur une échelle de 1 à 6 (tableau 1) pour les réponses aux questions du CSM-QA de l'ESCC de 2012. Les réponses moyennes sur une échelle de 6 points allaient de 3,78 pour le plus faible (à la question 6 , " notre société devient un meilleur endroit pour les gens comme vous ») à 5,44 pour le plus élevé (à la question 11, "vous avez des relations chaleureuses et fondées sur la confiance avec d'autres personnes »). Avec l'échelle originale, la normalité univariée des réponses était faible : l'aplatissement était supérieur à 3,0 pour 4 variables. En revanche, l'aplatissement et l'asymétrie étaient acceptables pour toutes les variables avec l'échelle fondée sur les jours. Les données manquantes constituaient moins de $2 \%$ des données de l'échantillon de l'ESCC de 2012, sauf pour 3 questions sur la sous-échelle du bien-être social. Pour l'une de ces questions (question 6 ), il manquait près de $5 \%$ des données $(4,8 \%)$. Nous disposions de données complètes pour les 14 questions pour $90 \%$ de l'échantillon.

Les niveaux de données manquantes étaient considérablement plus élevés pour les questions du CSM-QA de l'échantillon de l'ESCC de 2011-2012 : de 5,7\% à $14 \%$, avec des moyennes de réponses toutefois semblables. Les questions relatives à la sous-échelle du bien-être social offraient le pourcentage le plus élevé de données manquantes $(7,7 \%$ à $14 \%)$. Les corrélations entre les questions variaient de 0,58 à 0,62 pour la sous-échelle du bien-être émotionnel, de 0,28 à 0,55 pour la sous-échelle du bien-être social et de 0,36 à 0,51 pour la sous-échelle du bien-être psychologique (données non présentées). Les corrélations contenues dans ces intervalles signifient une bonne factorisabilité des questions. Le coefficient alpha de Cronbach s'approchait d'un niveau acceptable, à 0,82 pour la sous-échelle du bien-être émotionnel et 
TABLEAU 1

Moyennes, écarts-types et pourcentage de données manquantes par question, Continuum de santé mentale -

Questionnaire abrégé, Enquête sur la santé dans les collectivités canadiennes - Santé mentale de 2012

et Enquête sur la santé dans les collectivités canadiennes de 2011-2012, Canada, adultes âgés de 18 ans et plus

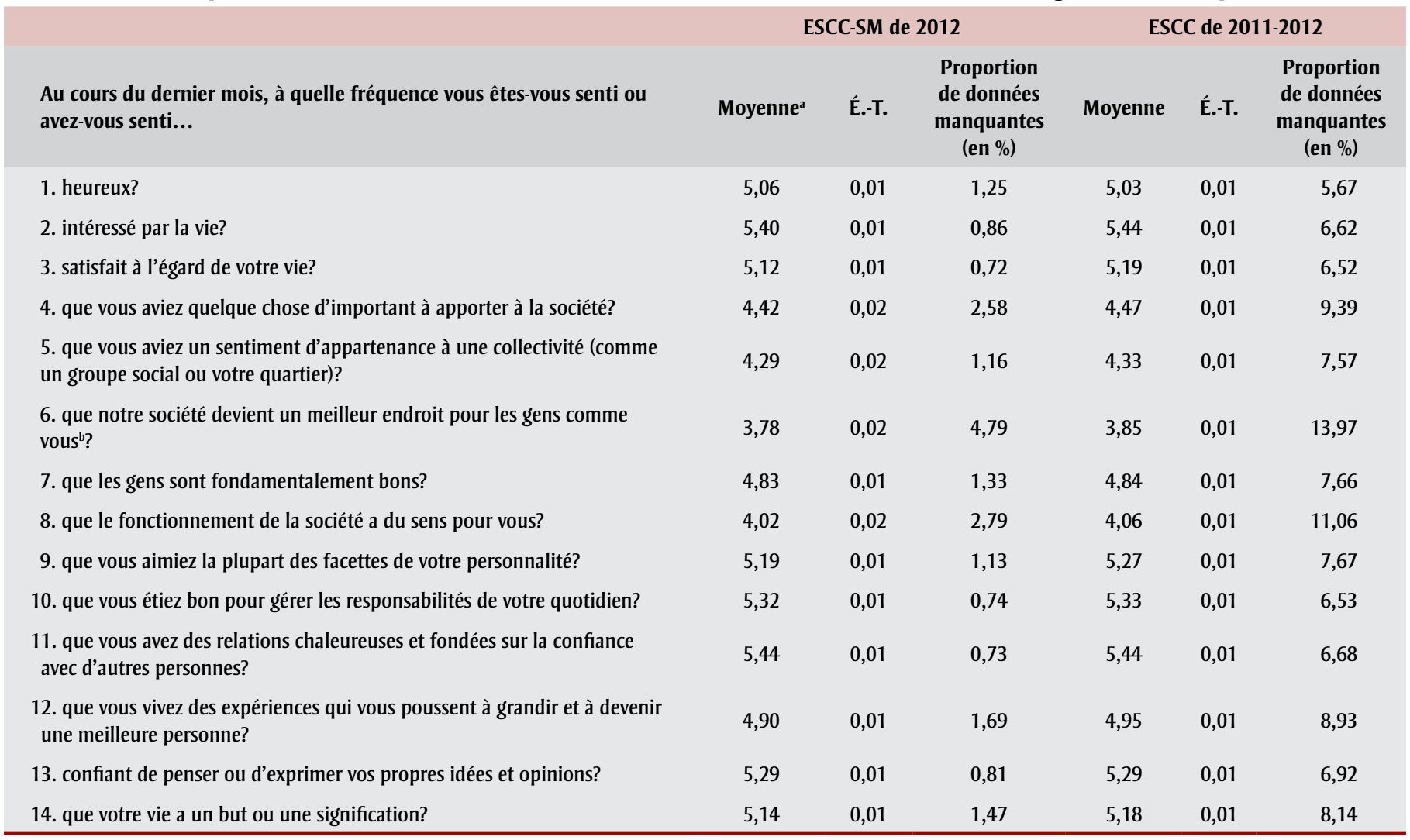

Abréviations : ESCC, Enquête sur la santé dans les collectivités canadiennes; ESCC-SM, Enquête sur la santé dans les collectivités canadiennes - Santé mentale; É.-T., Écart-type.

${ }^{a}$ Le minimum pour l'ensemble des variables est de 1 et le maximum de 6.

${ }^{\mathrm{b}}$ Keyes recommande maintenant de tenir compte d'une autre version de cette question : « notre société devient un meilleur endroit pour tout le monde »9.

pour la sous-échelle du bien-être psychologique et à 0,77 pour la sous-échelle du bien-être social.

Nous avons testé la structure à trois facteurs proposée par Keyes en réalisant une AFC et une estimation de VMR à l'aide du logiciel Mplus. Nous avons d'abord exécuté le modèle en utilisant l'échelle originale (1 à 6) (tableau 2, modèle 1a). L'ajustement de ce modèle était inadéquat. Nous avons ensuite exécuté le modèle en utilisant l'échelle représentant le nombre de jours par mois (tableau 2, modèle 1b). Si ce modèle présentait un meilleur ajustement que le premier, avec un ETA $(0,036)$ et une REQMN $(0,043)$ acceptables, l'IAC $(0,927)$ et l'ITL $(0,911)$ témoignaient tout de même d'un ajustement insuffisant du modèle.

Les indices de modification suggéraient que si nous permettions la covariance des résidus des questions, nous obtiendrions les meilleures améliorations possible en matière d'ajustement du modèle : 8 avec 6 , 8 avec 7, 7 avec 6 et 6 avec 5. Le modèle comprenant ces erreurs corrélées (tableau 2, modèle $2 \mathrm{a}$ ) offrait un ajustement adéquat du modèle : l'IAC, l'ITL, l'ETA et la REQMN étaient tous supérieurs aux seuils acceptés. Le critère d'information d'Akaike (CIA) diminuait de 3775,646 avec 4 degrés de liberté. Nous n'avons obtenu un ajustement adéquat pour ce modèle à trois facteurs qu'en ajoutant quatre termes d'erreur covariants impossibles à expliquer clairement d'un point de vue théorique. Ce modèle de base est présenté figure 1 .

Nous avons ajusté ce modèle à trois facteurs aux données de l'ESCC de 20112012 afin d'en confirmer la stabilité. Dans cet échantillon, le modèle incluant les termes d'erreur corrélés portant sur le facteur du bien-être social ont offert un ajustement adéquat (tableau 2, modèle 2b), ce qui laisse penser que ce modèle à trois facteurs est stable dans l'ensemble des échantillons.

Nous avons calculé une matrice de corrélation entre scores sur une échelle continue et concepts associés, comme l'illustre le tableau 3. La corrélation entre la souséchelle du bien-être émotionnel et celle du bien-être psychologique était de 0,62. Les deux sous-échelles présentaient des corrélations positives et significatives avec la satisfaction à l'égard de la vie, l'autoévaluation de la santé mentale, le sentiment d'appartenance à la communauté et l'Échelle de provisions sociales. La satisfaction à l'égard de la vie et l'auto-évaluation de la santé mentale étaient plus fortement corrélées au bien-être émotionnel (respectivement 0,57 et 0,47 ).

Les sous-échelles présentaient des corrélations négatives et significatives avec les 
TABLEAU 2

Résultats de l'analyse factorielle confirmatoire du Continuum de santé mentale - Questionnaire abrégé, Enquête sur la santé dans les collectivités canadiennes - Santé mentale de 2012 et Enquête sur la santé dans les collectivités canadiennes de 2011-2012, Canada

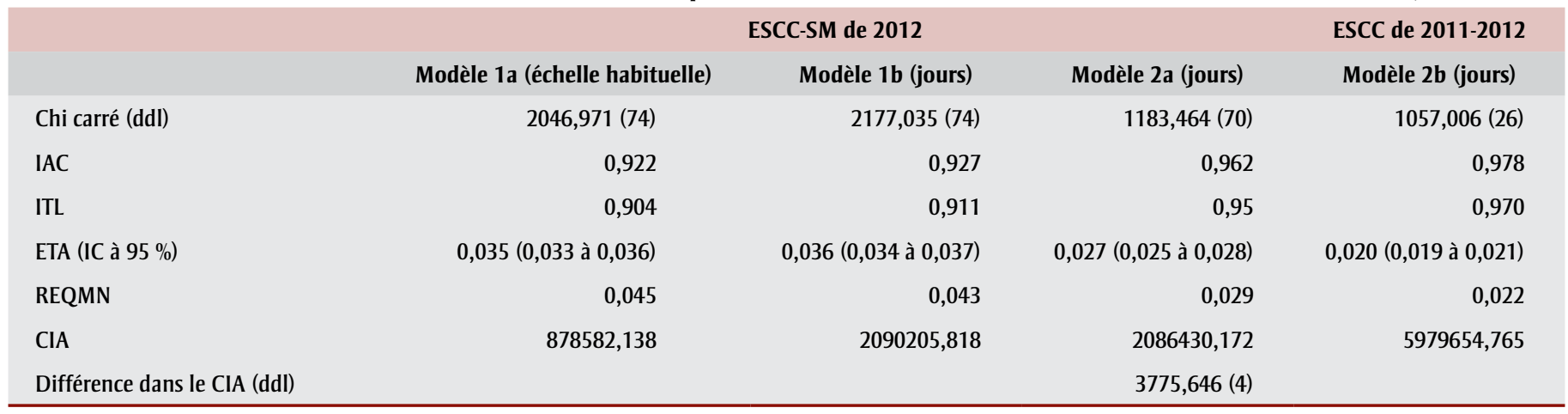

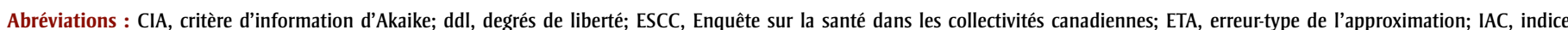
d'ajustement comparatif; IC, intervalle de confiance; ITL, indice de Tucker et Lewis; REQMN, erreur quadratique moyenne normalisée; SM, santé mentale.

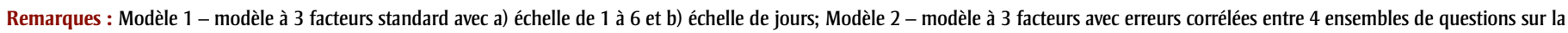
sous-échelle du bien-être social.

concepts de détresse psychologique, d'interactions sociales négatives et avec l'outil WHODAS d'évaluation des handicaps de l'OMS, les corrélations étant plus fortes entre les sous-échelles et la détresse psychologique qu'avec les interactions sociales négatives et l'outil WHODAS. Toutes les corrélations étaient significatives à un niveau prudent de $p<0,001$.

En revanche, l'échelle du bien-être social présentait une corrélation plus faible avec les facteurs associés que l'échelle du bienêtre émotionnel et celle du bien-être psychologique. En particulier, les corrélations entre l'échelle du bien-être social et les provisions sociales et les interactions sociales négatives étaient plus faibles que prévu,

FIGURE 1

Analyse factorielle confirmatoire du Continuum de santé mentale - Questionnaire abrégé, coefficients normalisés

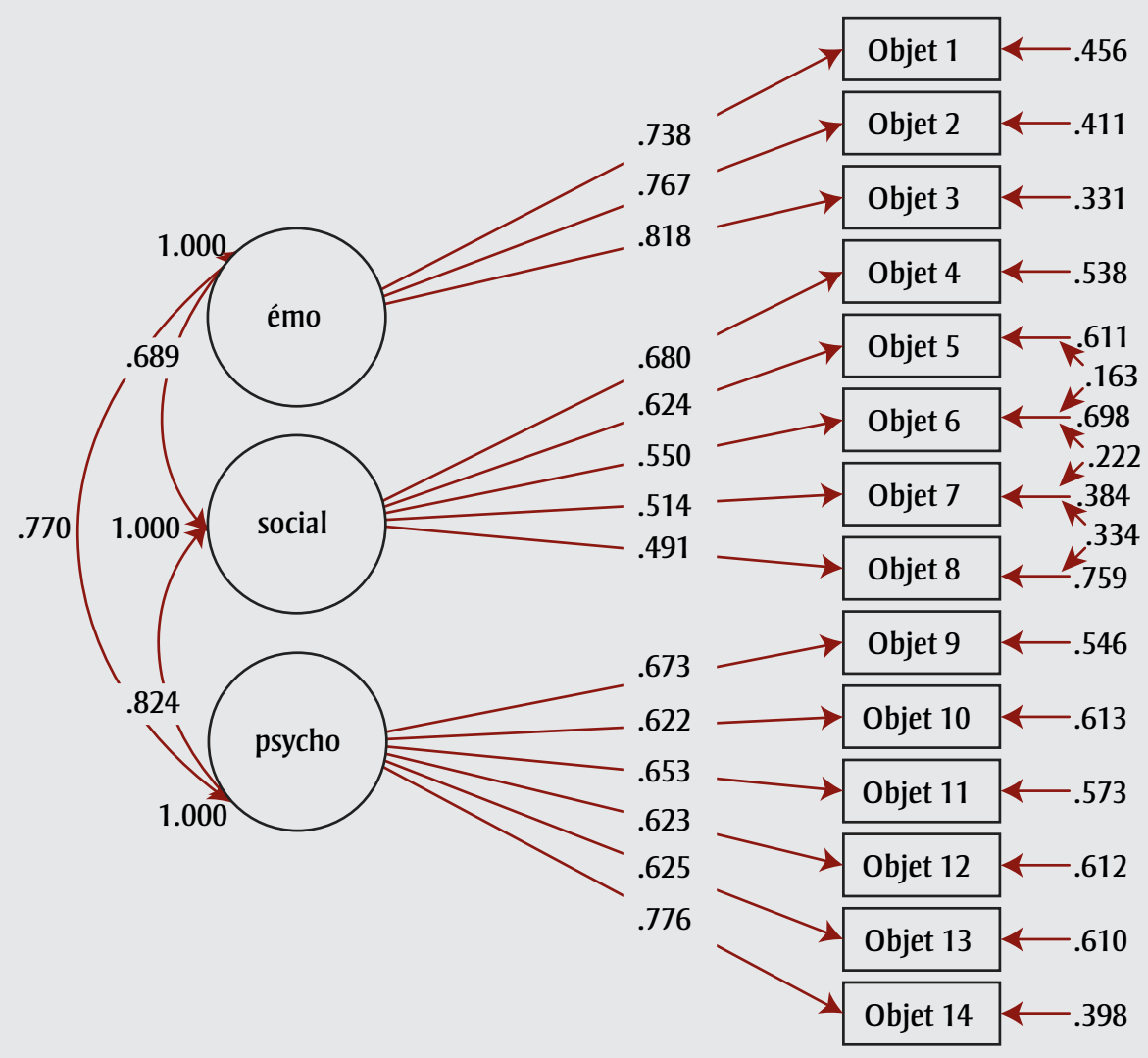

Abréviations : CSM-QA, Continuum de santé mentale - Questionnaire abrégé; émo, bien-être émotionnel; psycho, bien-être psychologique; social, bien-être social. Remarque : Les objets 1 à 14 se rapportent aux questions 1 à 14 du CSM-QA. 
TABLEAU 3

Corrélations des échelles de bien-être émotionnel, de bien-être psychologique et de bien-être social du Continuum de santé mentale Questionnaire abrégé et concepts associés, Enquête sur la santé dans les collectivités canadiennes - Santé mentale de 2012

\begin{tabular}{lccc} 
& Bien-être émotionnel & Bien-être psychologique & 0,49 \\
Bien-être émotionnel & 1,00 & 0,62 & 0,61 \\
Bien-être psychologique & 0,62 & 1,00 & 1,00 \\
Bien-être social & 0,49 & 0,61 & 0,34 \\
Satisfaction à l'égard de la vie & 0,57 & 0,42 & 0,29 \\
Auto-évaluation de la santé mentale & 0,47 & 0,27 & 0,40 \\
Sentiment d'appartenance & 0,24 & 0,37 & 0,28 \\
Échelle de provisions sociales & 0,37 & $-0,26$ & $-0,21$ \\
Interactions sociales négatives & $-0,27$ & $-0,47$ & $-0,36$ \\
Détresse psychologique & $-0,55$ & $-0,29$ & $-0,21$ \\
WHODAS & $-0,33$ & & 0 \\
\hline
\end{tabular}

Abréviations : CSM-QA, Continuum de santé mentale - Questionnaire abrégé; WHODAS, outil Disability Assessment Schedule de l’OMS.

Remarque : Toutes les corrélations sont significatives à une valeur de $p<0,001$.

soit respectivement 0,28 et $-0,21$. La corrélation avec le sentiment d'appartenance était modérée, au niveau prévu de 0,40.

\section{Analyse}

\section{Points forts et limites}

Cette étude avait pour objet la structure factorielle et les propriétés psychométriques du CSM-QA utilisé auprès des membres de ménages canadiens et examinait la validité reliée aux critères des scores obtenus sur les diverses échelles. L'AFC a confirmé la validité de la structure fondée théoriquement sur trois facteurs pour les membres adultes des ménages canadiens, que ce soit avec l'échelle originale (1 à 6) ou lorsque les réponses du CSM-QA allant de " jamais " à " tous les jours " étaient classées selon une échelle variant de 0 à 28 jours, dans la mesure cependant où nous ajoutions quatre termes d'erreur corrélés au modèle.

Tous les termes d'erreur corrélés relevaient de l'échelle du bien-être social, qui doit donc être utilisée avec prudence en combinaison avec plusieurs éléments de preuve (quantités plus élevées de données manquantes pour ce facteur, corrélations plus faibles avec les concepts associés). Ce fonctionnement moindre de l'échelle du bien-être social corrobore les conclusions d'autres études ${ }^{19}$. Nous suggérons d'utiliser les sous-échelles du bien-être émotionnel et du bien-être psychologique à titre de mesures de la santé mentale positive de la population canadienne, une mesure alternative du bien-être social étant peut-être nécessaire.

Nous avons examiné la validité reliée aux critères des sous-échelles du bien-être émotionnel, du bien-être social et du bienêtre psychologique en nous penchant sur les corrélations entre ces scores et un certain nombre de concepts dont nous avions pensé qu'ils seraient associés de manière soit positive, soit négative. Nous avons observé des corrélations positives et significatives avec les deux sous-échelles pour la satisfaction à l'égard de la vie, l'autoévaluation de la santé mentale et le sentiment d'appartenance à la communauté et les provisions sociales. Nous avons observé des corrélations négatives et significatives pour la détresse psychologique, les interactions sociales négatives et l'outil WHODAS d'évaluation des handicaps de l'OMS.

De façon générale, les concepts plus étroitement liés au bien-être émotionnel ou au bien-être psychologique (comme la satisfaction à l'égard de la vie dans le cas du bien-être émotionnel) présentaient des corrélations plus fortes que les concepts moins étroitement liés à ces facteurs (comme l'outil WHODAS d'évaluation des handicaps de l'OMS dans le cas du bienêtre psychologique). Ces conclusions renforcent la validité des critères des mesures.

Si d'autres mesures de la validité convergente auraient été idéales, comme l'estime de soi et la réalisation de soi pour le bien-être psychologique, nous étions limités par le contenu disponible dans les enquêtes utilisées pour ces analyses. Les recherches subséquentes sur le sujet pourraient se pencher à la fois sur la structure factorielle des mesures de la santé mentale et sur celle de la maladie mentale, comme l'ont fait Lamers et collab. ${ }^{15}$.

On doit également tenir compte des limites de l'analyse factorielle. Les décisions basées sur les indices de modification peuvent donner lieu à un modèle trop ajusté, c'està-dire incluant des termes sans signification de fond ou correspondant à des caractéristiques propres à un échantillon impossibles à valider de manière croisée. Étant donné que l'on ne peut ajouter de termes supplémentaires que lorsqu'il est possible de les expliquer théoriquement, nous avons plutôt choisi d'inclure les termes d'erreur covariants suggérés par les indices de modification, même s'ils sont difficiles à justifier d'un point de vue théorique.

Nous avons observé une baisse de la proportion de données manquantes entre la mise en œuvre du CSM-QA dans l'ESCC de 2011-2012 et dans l'ESCC-SM de 2012, ce qui pourrait signifier que le fonctionnement irrégulier de l'échelle (en comparaison avec son fonctionnement dans d'autres études) serait partiellement attribuable à la formation des enquêteurs. Il faudrait donc refaire ces analyses la prochaine fois que le CSM-QA sera utilisé dans le cadre d'une enquête canadienne à grande échelle. 


\section{Conclusion}

Les résultats de notre étude invitent à penser que si, dans l'ensemble, la validité conceptuelle du CSM-QA est renforcée par deux échantillons canadiens de grande taille, la prudence est de mise lorsque l'on utilise la sous-échelle du bien-être social dans le cadre d'enquêtes auprès de la population du Canada. D'autres recherches sont nécessaire afin de déterminer pourquoi cette sous-échelle n'a pas fonctionné aussi efficacement qu'avec d'autres échantillons, quelles sont les causes potentielles de cette différence de fonctionnement et quelles mesures de substitution sont à élaborer. À la lumière de ces conclusions, nous recommandons d'utiliser une mesure de substitution (comme le sentiment d'appartenance à la communauté) pour rendre compte du bien-être social de la population canadienne adulte, et ce, jusqu'à ce qu'une mesure plus précise soit élaborée et testée. Les recherches à venir devraient tenter de déterminer si la validité de la méthode présentée dans notre article est la même avec d'autres populations et dans le cadre d'autres études.

\section{Remerciements}

Nous aimerions remercier les pairs évaluateurs du présent article pour les suggestions utiles qu'ils nous ont soumises. Nous tenons également à remercier Louise McRae des commentaires fournis au sujet de ce projet au cours de son élaboration.

\section{Conflits d'intérêts}

Les auteurs n'ont aucun conflit d'intérêts à déclarer.

\section{Contributions des auteurs}

HO, JV, JD et GJ ont contribué à la conception de l'étude. HO a analysé les données. HO, JV, JD et GJ ont interprété les données. HO a élaboré l'ébauche du manuscrit. HO, JV, JD et GJ ont relu et révisé le manuscrit. Tous les auteurs ont lu et donné leur approbation que la version finale de cet article soit publiée, et étaient d'accord de se porter garants du travail.

\section{Références}

1. Commission de la santé mentale du Canada. La nécessité d'investir dans la santé mentale au Canada. Ottawa (Ont.) : Commission de la santé mentale du Canada; 2013
2. Joubert N, Raeburn J. Mental health promotion : people, power and passion, Int J Ment Health Promot. 1998; $1: 15-22$.

3. Organisation mondiale de la santé. Dix faits sur la santé mentale [Internet]. Genève (Suisse) : Organisation mondiale de la santé [mise à jour en août 2014; consultation le 26 septembre 2015]. En ligne à : http://www .who.int/features/factfiles/mental _health/fr/

4. Tennant R, Hiller L, Fishwick R, et al. The Warwick-Edinburgh mental wellbeing scale (WEMWBS): development and UK validation. Health Qual Life Outcomes. 2007;5(1):63. doi: 10.1186 /1477-7525-5-63.

5. Diener E, Wirtz D, Tov W, et al. New well-being measures: short scales to assess flourishing and positive and negative feelings. Soc Indic Res. 2010; 97(2):143-156.

6. Huppert FA, So TT. Flourishing across Europe: application of a new conceptual framework for defining wellbeing. Soc Indic Res. 2013;110(3): 837-861.

7. Seligman M. S'épanouir : pour un nouvel art du bonheur. Paris : Belfond 2013.

8. Keyes CLM. The mental health continuum: from languishing to flourishing in life. J Health Soc Behav. 2002;42(2): 207-222.

9. Keyes CLM. Atlanta : Brief description of the mental health continuum short form (MHC-SF) [Internet]. [2009, consultation le 25 octobre 2015]. En ligne à : https://www.aacu.org/sites/default /files/MHC-SFEnglish.pdf

10. Bradburn NM. The structure of psychological well-being. Chicago : Aldine; 1969. 320 pages.

11. Cantril H. The pattern of human concerns. New Brunswick (NJ) : Rutgers University Press; 1965. 427 pages.

12. Ryff CD. Psychological well-being in adult life. Curr Dir Psychol Sci. 1995; 4(4):99-104.
13. Keyes CLM. Social well-being. Soc Psychol Q. 1998;61(2):121-140.

14. Keyes CLM, Wissing M, Potgieter JP, Temane M, Kruger A, van Rooy S. Evaluation of the Mental Health Continuum-Short Form (MHC-SF) in Setswana-speaking South Africans. Clin Psychol Psychother. 2008;15(3): 181-192.

15. Lamers SM, Westerhof GJ, Bohlmeijer ET, ten Klooster PM, Keyes CL. Evaluating the psychometric properties of the Mental Health ContinuumShort Form (MHC-SF). J Clin Psychol. 2011;67(1):99-110.

16. Joshanloo M, Wissing MP, Itumeleng $\mathrm{PH}$, Lamers SM. Measurement invariance of the Mental Health ContinuumShort Form (MHC-SF) across three cultural groups. Pers Individ Differ. 2013;55(7):755-759.

17. Keyes CL, Eisenberg D, Perry GS, Dube SR, Kroenke K, Dhingra SS. The relationship of level of positive mental health with current mental disorders in predicting suicidal behavior and academic impairment in college students. J Am Coll Health. 2012;60(2): 126-133.

18. Keyes CL. Mental health in adolescence: is America's youth flourishing? Am J Orthopsychiatry. 2006;76(3): 395-402.

19. Petrillo G, Capone V, Caso D, Keyes CL. The Mental Health ContinuumShort Form (MHC-SF) as a measure of well-being in the Italian context. Soc Indic Res. 2014;121(1):291-312.

20. Gilmour H. Santé mentale et maladie mentale. Rapports sur la santé. 2014; 25(9):3-10.

21. Janda LH. Psychological testing : theory and applications. Boston (MA) : Allyn and Bacon; 1998.

22. Statistique Canada. Enquête sur la santé dans les collectivités canadiennes - Santé mentale [Internet]. Ottawa (Ont.) : Statistique Canada; [mise à jour le 10 septembre 2013, consultation le 26 septembre 2015]. En ligne à : http://www23.statcan.gc .ca/imdb-bmdi/pub/5015-fra.htm 
23. Statistique Canada. Enquête sur la santé dans les collectivités canadiennes - Composante annuelle de 2011-2012 [Internet]. Ottawa (Ont.) : Statistique Canada; [mise à jour le 12 juin 2012, consultation le 26 septembre 2015]. En ligne à : http:// www23.statcan.gc.ca/imdb/p2SV_f .pl? Function $=$ getSurvey $\& I d=114112$

24. OCDE. OECD guidelines on measuring subjective well-being. Paris : OECD Publishing; 2013. [n ${ }^{\circ}$ KE-31-13-501EN-C]

25. Cutrona CE, Russell DW. The provisions of social relationships and adaptation to stress. Advances Personal Relatsh. 1987;(1)1:37-67.

26. Cairney J, Veldhuizen S, Wade TJ, Kurdyak P, Streiner DL. Evaluation of 2 measures of psychological distress as screeners for depression in the general population. Can J Psychiatry. 2007;52(2):111-120.

27. Üstün TB, Kostanjsek N, Chatterji S, Rehm J, editors. Measuring health and disability: manual for WHO disability assessment schedule WHODAS 2.0. Genève : Organisation mondiale de la santé; 2010.

28. Krause N. Negative interaction and satisfaction with social support among older adults. J Gerontol B Psychol Sci Soc Sci. 1995;50B(2): P59-P73.

29. Hu L, Bentler PM. Cutoff criteria for fit indexes in covariance structure analysis: conventional criteria versus new alternatives. Struct Equ Model. 1999; 6(1):1-55. 